\title{
Adventures in Personalized Information Access ${ }^{\star}$
}

\author{
Barry Smyth \\ Adaptive Information Cluster, School of Computer Science and Informatics, \\ University College Dublin, Ireland \\ barry. smyth@ucd.ie
}

\begin{abstract}
Access to information plays an increasingly important role in our everyday lives and we have come to rely more and more on a variety of information access services to bring us the right information at the right time. Recently the traditional one-size-fits-all approach, which has informed the development of the majority of today's information access services, from search engines to portals, has been brought in to question as researchers consider the advantages of more personalized services. Such services can respond to the learned needs and preferences of individuals and groups of like-minded users. They provide for a more proactive model of information supply in place of today's reactive models of information search. In this talk we will consider the key challenges that motivate the need for a new generation of personalized information services, as well as the pitfalls that lie in wait. We will focus on a number of different information access scenarios, from e-commerce recommender systems and personalized mobile portals to community-based web search. In each case we will describe how different machine learning and data mining ideas have been harnessed to take advantage of key domain constraints in order to deliver information access interfaces that are capable of adapting to the changing needs and preferences of their users. In addition, we will describe the results of a number of user studies that highlight the potential for such technologies to significantly enhance the user experience and the ability of users to locate relevant information quickly and reliably.
\end{abstract}

* Invited speakers at ECML/PKDD are supported by the PASCAL European network of excellence. 